\title{
miR-185 and miR-29a are similarly expressed in the bronchoalveolar lavage cells in IPF and lung cancer but common targets DNMT1 and COL1A1 show disease specific patterns
}

\author{
ELENI BIBAKI ${ }^{1,2}$, ELIZA TSITOURA ${ }^{1}$, EIRINI VASARMIDI ${ }^{1,2}$, GEORGE MARGARITOPOULOS $^{1}$, \\ ATHINA TRACHALAKI ${ }^{1}$, CHARA KOUTOULAKI $^{1}$, THEODORA GEORGOPOULOU ${ }^{1,2}$, \\ DEMETRIOS A. SPANDIDOS ${ }^{3}$, NIKOS TZANAKIS ${ }^{2}$ and KATERINA M. ANTONIOU ${ }^{1,2}$ \\ ${ }^{1}$ Laboratory of Molecular and Cellular Pneumonology, Medical School, University of Crete; ${ }^{2}$ Department of \\ Thoracic Medicine, Interstitial Lung Disease Unit, University Hospital, University of Crete, 71110 Heraklion; \\ ${ }^{3}$ Laboratory of Clinical Virology, Medical School, University of Crete, 71003 Heraklion, Crete, Greece
}

Received February 1, 2018; Accepted March 19, 2018

DOI: $10.3892 / \mathrm{mmr} .2018 .8778$

\begin{abstract}
Idiopathic pulmonary fibrosis (IPF) and lung cancer (LC) constitute two progressively devastating lung diseases with common risk factors including aging and smoking. There is an increasing interest in the investigation of common pathogenic mechanisms between IPF and LC with therapeutic implications. Several oncomirs, microRNAs associated with malignancy, are also linked with IPF. miR-29a and miR-185 downregulation is probably involved both in carcinogenesis and fibrogenesis. We have previously observed miR-29a and miR-185 downregulation in IPF cells from bronchoalveolar lavage (BAL) and in this study we investigated their expression in LC BAL cells. Common targets of miR-29a and miR-185 such as DNA methyltransferase (DNMT)1, DNMT3b, COL1A1, AKT1 and AKT2 were measured. Potential correlations with pulmonary function tests, smoking status and endobronchial findings were investigated. Similar levels of miR-29a and miR-185 were detected in IPF and LC while their common targets AKT1 and DNMT3b were not found to differ, suggesting potential pathogenetic similarities at the level of
\end{abstract}

Correspondence to: Dr Katerina M. Antoniou, Laboratory of Molecular and Cellular Pneumonology, Medical School, University of Crete, 71110 Heraklion, Crete, Greece

E-mail: kantoniou@med.uoc.gr

Abbreviations: DNMT, DNA methyltransferase; IPF, idiopathic pulmonary fibrosis; LC, lung cancer; HRCT, high resolution computed tomography; $\mathrm{FEV}_{1}$, forced expiratory volume in $1 \mathrm{sec}$; $\mathrm{FVC}$, forced vital capacity, $\mathrm{DL}_{\mathrm{CO}}$, diffusing capacity for carbon monoxide; BAL, bronchoalveolar lavage; PFTs, pulmonary function tests

Key words: idiopathic pulmonary fibrosis, lung cancer, microRNAs, DNA methyltransferase, COL1A1 key epigenetic regulators. By conrast, COL1A1 mRNA levels were increased in IPF suggesting a disease-specific mRNA signature. Notably, DNMT1 was downregulated in the LC group and its expression was further reduced in the presence of increasing malignant burden as it was implied by the endobronchial findings.

\section{Introduction}

Idiopathic pulmonary fibrosis (IPF) is a form of chronic progressive interstitial pneumonia that continues to be associated with high morbidity and mortality, despite the fact that new anti-fibrotic drugs have recently been added to our quiver (1).

The exact cause of the disease remains unknown, however there is a strong association with aging and smoking, similarly to lung cancer (LC) (2,3). Aggregating mutational burden, increased epigenetic gene silencing through aberrant DNA methylation patterns and telomere dysfunction frame the probable underlying correlation (4-8). A deeper view of the pathophysiology of the two diseases reveals several similarities and common pathways involved (4,5,9-11). Crucial cellular mechanisms known to be involved in cell proliferation, resistance to apoptosis and epithelial-mesenchymal transition, are activated in both LC and pulmonary fibrosis (12-14). Preneoplastic lesions (atypia, metaplasia, dysplasia), microsatellite instability and loss of heterozygosity in genes that underlie the tumorigenesis have also been reported in lung fibrosis tissue (15).

Interestingly, $\mathrm{LC}$ is a common comorbidity among patients with IPF with major impact on their survival. Increasing data support the hypothesis that LC occurs secondarily on the ground of fibrosis rather than as a preceding finding. In the majority of patients, LC arise as nodular lesions in the peripheral area of fibrosis, mainly at the lower areas of the lungs with squamous cell and adenocarcinoma being the prevalent histological types $(12,16)$.

MicroRNAs have emphatically emerged as major regulators of gene expression through epigenetic and post-transcriptional 
mechanisms. MicroRNA profiling studies in LC have currently proposed several microRNAs as potential epigenetic biomarkers in the diagnostic procedure. Similarly, in IPF several oncomirs are consistently deregulated, such as the miR-29 family (17). The miR-29 family is usually downregulated in affected tissues and plays a crucial role in fibrogenesis in multiple organs including lung (18), liver (19) and kidney (20). Among the targets of miR-29 are collagens, enzymes required for collagen synthesis $(21,22)$, matrix metalloproteases and DNA methylation enzymes such as DNA methyltransferase (DNMT)3A/B (23). The miR-29 family can be an effective regulator of tumorigenesis and cancer progression by targeting multiple tumor-related pathways as cell proliferation, cell cycle, apoptosis and metastasis as well as affecting the epigenetic and immune regulation (24).

Additionally, miR-185 is increasingly recognized as an oncomir commonly deregulated in IPF and LC (25). Downregulation of miR-185 has been demonstrated in IPF lung tissue (26) and in several malignancies including LC, glioma, hepatocellular and breast cancer (27). miR-185 supplementation inhibits cell growth and proliferation by directly targeting AKT1 in NSCLC28, through DNMT1 targeting, leading to the PTEN upregulation and inhibition of AKT phosphorylation (29).

Interestingly, the expression of miR-29 and miR-185 is downregulated by TGFb $(18,25,30)$. Furthermore, there are emerging data suggesting an overlap of miR-29a and miR-185 targets; miR-185 appears to regulate collagens $(26,30,31)$, while miR-29a seems to regulate AKT1 (32) and AKT2 (33). Recently, both miR-185 and miR-29a were downregulated in IPF bronchoalveolar lavage (BAL) cells compared to controls (25).

BAL is becoming an attractive, minimally invasive tool to study alveolar macrophages and other immune cells, as well as other lung resident cells that may derive from adjacent lesions. In essence BAL could be considered a form of liquid biopsy in LC and possibly in IPF34. In this study, the expression of miR-29a and miR-185 was compared in IPF and LC. Well-established targets of miR-29a and recently emerged miR-185 targets such as DNMTs (DNMT1 and DNMT3b), AKT1/AKT2 and COL1A1 were analyzed. Furthermore, microRNA and target gene expression was evaluated in the LC group according to LC type and endobronchial findings, including, the presence of endobronchial lesion(s), the side of the BAL procedure relative to the tumor site and the cytology findings for malignant cells.

In our study, similar levels of miR-29a, miR-185 and their targets AKT1, AKT2 and DNMT3b were found in the two diseases. By contrast, COL1A1 mRNA levels were increased in IPF suggesting a disease-specific mRNA signature. Importantly, DNMT1 was downregulated in the LC group and its expression was further reduced in the presence of increasing malignant burden as indicated by the endobronchial findings further suggesting an LC-specific signature.

\section{Materials and methods}

Human subjects. Patients were classified as ever smokers and non-smokers. Pulmonary function tests (PFTs), performed within 1 month of $\mathrm{CT}$, included forced expiratory volume in $1 \mathrm{sec}\left(\mathrm{FEV}_{1}\right)$, forced vital capacity $(\mathrm{FVC})$, and diffusing capacity for carbon monoxide $\left(\mathrm{DL}_{\mathrm{CO}}\right)$ corrected for hemoglobin concentration, expressed as percentages of the predicted normal values. A total of 89 subjects were enrolled in this study, comprising patients with IPF $(n=57)$ and patients with LC ( $n=32)$ with the majority being diagnosed as NSCLC $(n=29)$. All subjects were recruited from the Department of Thoracic Medicine, University Hospital of Heraklion (Crete, Greece) between December 2013 and July 2017. The study was approved by the Ethics Committee of the University Hospital of Heraklion (IRB no. 17030/12-12-2013 for IPF patients and Reg. no. 140/4-2-2015 for LC patients). All the patients provided informed consent in written form.

IPF group: The IPF patients were evaluated with complete PFTs, performed within 1 month of CT, including spirometry, measurement of lung volumes and diffusion capacity. Spirometry, lung volumes using the helium-dilution technique and $\mathrm{DL}_{\mathrm{CO}}$ (corrected for haemoglobin) using the single breath technique were performed using a computerized system (Jaeger 2.12; MasterLab, Würzburg, Germany). Predicted values were obtained from the standardized lung function testing of the European Coal and Steel Community, Luxembourg (1993). PFTs, $\mathrm{FEV}_{1}, \mathrm{FVC}$, and $\mathrm{DL}_{\mathrm{CO}}$ corrected for haemoglobin concentration, and were expressed as percentages of the predicted normal values. The diagnosis of IPF was based on open or video-assisted thoracoscopic biopsy, with all the biopsies reviewed by the same two histopathologists, or using ATS/ERS clinical and high resolution computed tomography (HRCT) criteria (35). In accordance with the aforementioned criteria, any known cause of pulmonary fibrosis, such as a systemic connective tissue disorder, was excluded by immunologic screening and rheumatologic clinical evaluation (35). All the IPF patients were newly diagnosed and had not received previous treatment.

LC group: The diagnosis of LC patients and the exact histological type of malignancy were based on lung biopsy proceeded through bronchoscopy, CT-guided biopsy by invasive radiologists or open lung biopsy. The LC patients were all chemo/radio/immunotherapy naive patients and their diagnostic management and staging were based on the International Association for the Study of Lung Cancer (36). The group of LC patients was further divided into subgroups associated with endobronchial findings evaluated during bronchoscopy and following BAL cytology evaluation. Thus, LC patients were assigned into groups according to the side of the BAL procedure relative to the side of the malignant lesion as it was depicted in the CT scan with a group termed 'same side' (SS) and a group of 'opposite side' (OS), if this separation was feasible, as patients with mediastinal lymphadenopathy could not fit in any of those groups. Secondly, the patients were separated in two groups according to the presence or absence of malignant cells in the BAL following cytology evaluation, termed 'negative cytology' (NC) and 'positive cytology' (PC) respectively. A third option for the cytology test was 'suspective for malignancy'. Finally, the patients were separated according to the physician's observations during bronchoscopy for obvious endobronchial lesions with a group termed 'endobronchial lesion' (OEL) and 'no endobronchial lesion' (NOEL). PFTs for LC patients were not included in the studies. 
Table I. Patient demographics.

\begin{tabular}{lccc}
\hline Demographics & LC $(32)$ & IPF $(\mathrm{n}=57)$ & P-value \\
\hline Age & $67.7( \pm 11.9)$ & $72( \pm 7.3)$ & 0.01 \\
Sex (M/F) & $26 / 6$ & $46 / 11$ & $\mathrm{NS}$ \\
Pyrs & $75(47.5-100)$ & $30(6-45)$ & 0.001 \\
Non smoker/ & $2 / 26$ & $13 / 43$ & NS \\
smoker & & & \\
PFTs & LC & IPF & \\
FVC, $\%$ & ND & $78.6( \pm 17,6)$ & NA \\
FEV,$\%$ & ND & $84.8( \pm 19.3)$ & NA \\
FEV $/$ FVC, $\%$ & ND & $84.21(79.8-89)$ & NA \\
TLC & ND & $75.5(62.1-83.4)$ & NA \\
TLCO/SB & ND & $51.3( \pm 16.8)$ & NA \\
KCO & ND & $88( \pm 23.7)$ & NA \\
BAL, $\%$ & LC & IPF & \\
Macrophages & $86.2(70.3-93.3)$ & $80.38(66-88)$ & NS \\
Lymphocytes & $5.8(3.6-11.1)$ & $6.4(3.5-12.7)$ & NS \\
Neutrophils & $3(1.3-6.3)$ & $5.8(2.4-12.9)$ & NS \\
Eosinophils & $0(0-1.5)$ & $1.1(0.4-2.6)$ & 0.01 \\
\hline
\end{tabular}

LC, lung cancer; IPF, idiopathic pulmonary fibrosis; M, male; F, female; PFTs, pulmonary function tests; FVC, forced vital capacity; $\mathrm{FEV}_{1}$, forced expiratory volume in $1 \mathrm{sec}$; BAL, bronchoalveolar lavage; TLC, total lung capacity; KCO, transfer coefficient of carbon monoxide; NS, not significant; NA, not applicable.

BAL cell isolation and determination of cellular composition. BAL was obtained from all the patients, as previously described (25). Cells (1-1.5 million) were homogenised in TriReagent $^{\mathrm{TM}}$ (MBL) for total RNA analysis, followed by storage at $-80^{\circ} \mathrm{C}$. Differential cell population count was analysed following May-Grünwald Giemsa staining of cell cytospins, as previously described (25).

MicroRNA and mRNA expression analyses. Total RNA was isolated as previously described (25) using the mirVana ${ }^{\mathrm{TM}}$ miRNA isolation kit (Ambion; Thermo Fisher Scientific, Inc., Waltham, MA, USA). For the analysis of microRNA expression levels, $10 \mathrm{ng}$ of total RNA were used in reverse transcriptase and quantitative PCR reactions using the TaqMan ${ }^{\mathrm{TM}}$ microRNA assays (Life Technologies; Thermo Fisher Scientific, Inc.) and 7500 Fast Real-Time PCR system (Applied Biosystems; Thermo Fisher Scientific, Inc.). For gene expression analyses, $500 \mathrm{ng}$ of total RNA were treated with DNAfree (Ambion; Thermo Fisher Scientific, Inc.) for genomic DNA contamination removal, followed by first-strand cDNA synthesis using Maxima RT ${ }^{\mathrm{TM}}$ (Fermentas; Thermo Fisher Scientific, Inc.) and qPCR analysis using Maxima SYBR-Green qPCRmix (Fermentas; Thermo Fisher Scientific, Inc.) on Mx3005P qPCR system (Agilent Technologies, Inc., Santa Clara, CA, USA). Probe and primer sequences are summarized in Table II. RNU19 levels were used as endogenous controls for the normalization of microRNA expression levels in BAL samples. GAPDH levels were used as endogenous control for the normalization of mRNA expression levels in BAL samples.
Table II. IDs of the TaqMan microRNA assays and primer sequences used for the quantification of microRNAs and mRNAs, respectively.

\begin{tabular}{lc} 
A, \\
\hline MicroRNA assay name & MicroRNA assay ID \\
\hline RNU19 & 001003 \\
hsa-miR-185 & 002271 \\
hsa-miR-29a & 002112
\end{tabular}

B,

Gene name Primer sequences

\begin{tabular}{|c|c|}
\hline GAPDH & $\begin{array}{l}\text { F: agccacatcgctcagaca } \\
\text { R: ccaatacgaccaaatccgtt }\end{array}$ \\
\hline COL1A1 & $\begin{array}{l}\text { F: gggattccctggacctaaag } \\
\text { R: ggaacacctcgctctcca }\end{array}$ \\
\hline$A K T 1$ & $\begin{array}{l}\text { F: gcagcacgtgtacgagaaga } \\
\text { R: ggtgtcagtctccgacgtg }\end{array}$ \\
\hline$A K T 2$ & $\begin{array}{l}\text { F: ctcacacagtcaccgagagc } \\
\text { R: tgggtctggaaggcatactt }\end{array}$ \\
\hline DNMT1 & $\begin{array}{l}\mathrm{F}: \text { tttctgatgaaaaagacgaggat } \\
\mathrm{R}: \text { tttctccgttggttctttgg }\end{array}$ \\
\hline DNMT3b & $\begin{array}{l}\text { F: agagggacatctcacggttc } \\
\text { R: ggttgccccagaagtatcg }\end{array}$ \\
\hline
\end{tabular}

DNMT, DNA methyltransferase.

Relative expression values per sample for each microRNA or mRNA assay were calculated by the $2^{-\Delta \Delta \mathrm{Cq}}$ method using as calibrator sample the average of DCT values.

Statistical analysis. Analysis of expression values with lung function tests and BAL cell population percentages was performed Prism 6 software. Group comparisons were made by analysis of variance, log transformation and Mann-Whitney test. Student's t-test, Wilcoxon rank-sum test, or Chi-square test were also used for comparisons as appropriate. $\mathrm{P}<0.05$ was considered statistically significant.

\section{Results}

Patient demographics. Demographic data and PFTs are summarised in Table I. IPF patients were older than the patients in the LC group by $4.3( \pm 4.6)$ years, while LC patients were heavier smokers as could be expected. The majority of LC was NSCLC (29/32), with the rest being SCLC. The group of LC patients was subdivided according to the endobronchial findings as described in materials and methods. Fourteen LC patients were assigned to the group SS and 9 to OS according to the site of broncoscopy. Fourteen LC patients had no malignant cells detected in their BAL and were assigned to the NC group and 7 to the PC. Nineteen patients had obvious OEL and 7 were assigned to NOEL. 
A

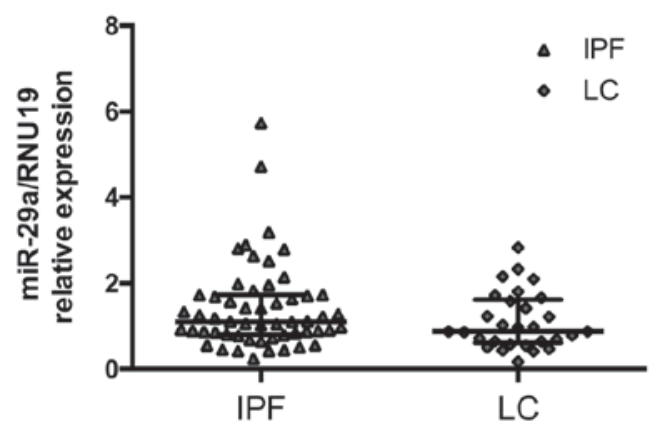

B

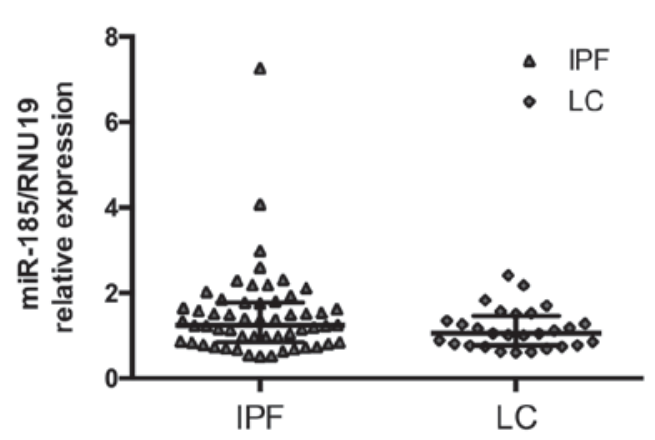

Figure 1. miR-29a and miR-185 levels are similar between IPF and lung cancer groups. (A) miR-29a and (B) miR-185 expression levels normalised by RNU19 in BAL cells from IPF and LC patients as shown by dot plots with median and interquartile range. IPF, idiopathic pulmonary fibrosis; BAL, bronchoalveolar lavage; LC, lung cancer.

A

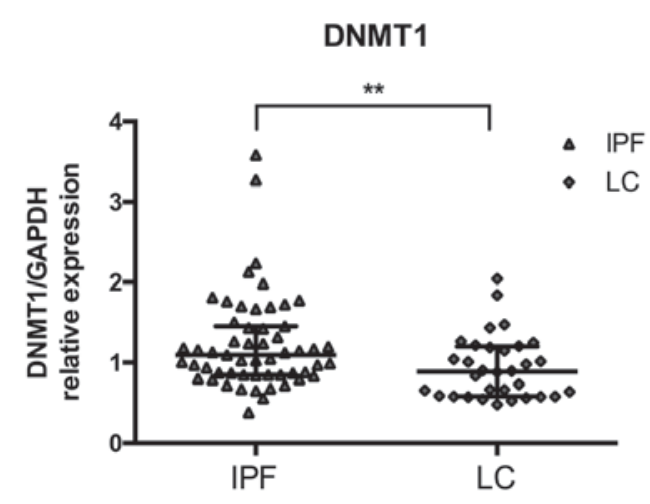

B

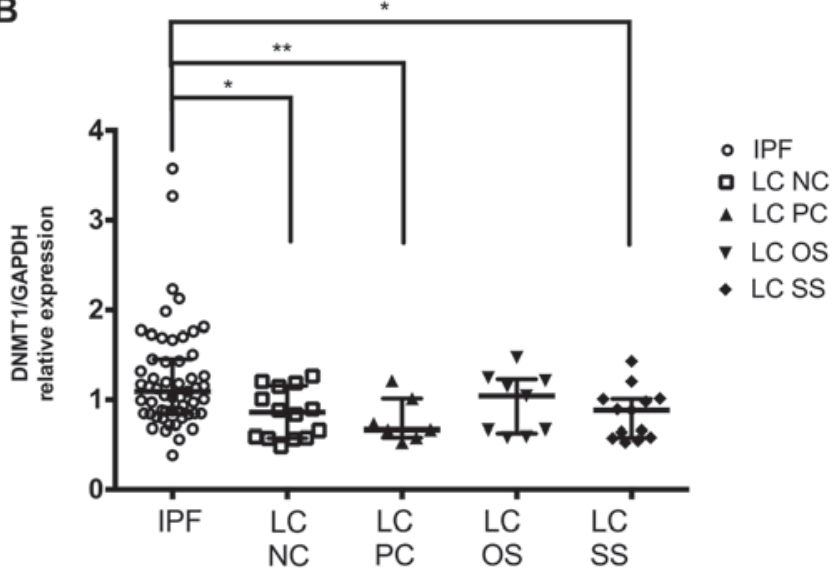

Figure 2. DNMT1 levels in LC patients compared to IPF patients. DNMT1 mRNA levels in BAL cells as measured by RT-qPCR and normalised using GAPDH (A) in LC group and (B) in LC subgroups NC, PC, OS and SS as compared to IPF. ${ }^{*} \mathrm{P}<0.05$, ${ }^{* *} \mathrm{P}<0.01$ of Mann-Whitney test. DNMT, DNA methyltransferase; LC, lung cancer; IPF, idiopathic pulmonary fibrosis; BAL, bronchoalveolar lavage; NC, negative cytology; PC, positive cytology; OS, opposite side; SS, same side.

miR-185 and miR-29a levels are similar between IPF and LC. MicroRNA expression levels in BAL cells were measured by RT-qPCR and normalized using small nucleolar RNA RNU19. We have previously shown that both microRNAs were significantly downregulated in IPF relative to controls (25). The downregulation of the two microRNAs was also noted for LC since the expression of miR-29a and miR-185 did not differ between IPF and LC patients (Fig. 1A and B). miR-29a and miR-185 expression levels were significantly correlated within the IPF group (Spearman's R 0.81, $\mathrm{P}=6 \mathrm{e}^{-14}$ ) and the LC group (Spearman's R 0.71, P=3.6e $\mathrm{e}^{-5}$ ).

DNMTs/AKT/miR-29a/miR-185 axis in IPF and LC. DNMT1 and DNMT3b are common targets of miR-185 and miR-29 in IPF and LC. mRNA expression levels in BAL cells were measured by RT-qPCR and normalized using GAPDH. DNMT1 levels previously measured in IPF relative to controls showed no differences. Notably, DNMT1 levels were significantly lower in LC patients compared to IPF patients. (Fig. 2A; Table III). No differences were detected in the levels of DNMT3b between the two diseases (Table III). No difference was detected in the levels of AKT1 and AKT2 between the two diseases (Table III).
Effect of malignant burden on DNMT1 levels in BAL cells compared to IPF. Comparing IPF patients with the LC group in detail, further reduced levels of DNMT1 were detected in the samples where the BAL procedure was performed at the side of the lesion (SS) as opposed to the lesion free side (OS). Moreover, LC patients with positive BAL cytology results (PC) had a more pronounced reduction in DNMT1 levels than those without the presence cells with malignant features (NC), when compared with IPF (Fig. 2B). Regarding the histological type of LC, the more obvious reduction in DNMT1 mRNA levels compared with IPF was found in NSLC type.

COL1A1/miR-29a/miR-185 axis in IPF and LC. Next we analysed miR-29a specific target COL1A1 by RT-qPCR and normalized using GAPDH. Our previous result showed that IPF BAL cells express significantly higher levels of COL1A1 mRNA than controls. In this study, we observed that the levels of COL1A1 mRNA were significantly lower in LC patients compared with IPF patients (Fig. 3A; Table III).

Effect of malignant burden on COL1Al levels in BAL cells compared to IPF. The mRNA levels of COL1A1 were markedly reduced in LC patients compared with IPF overall as it 


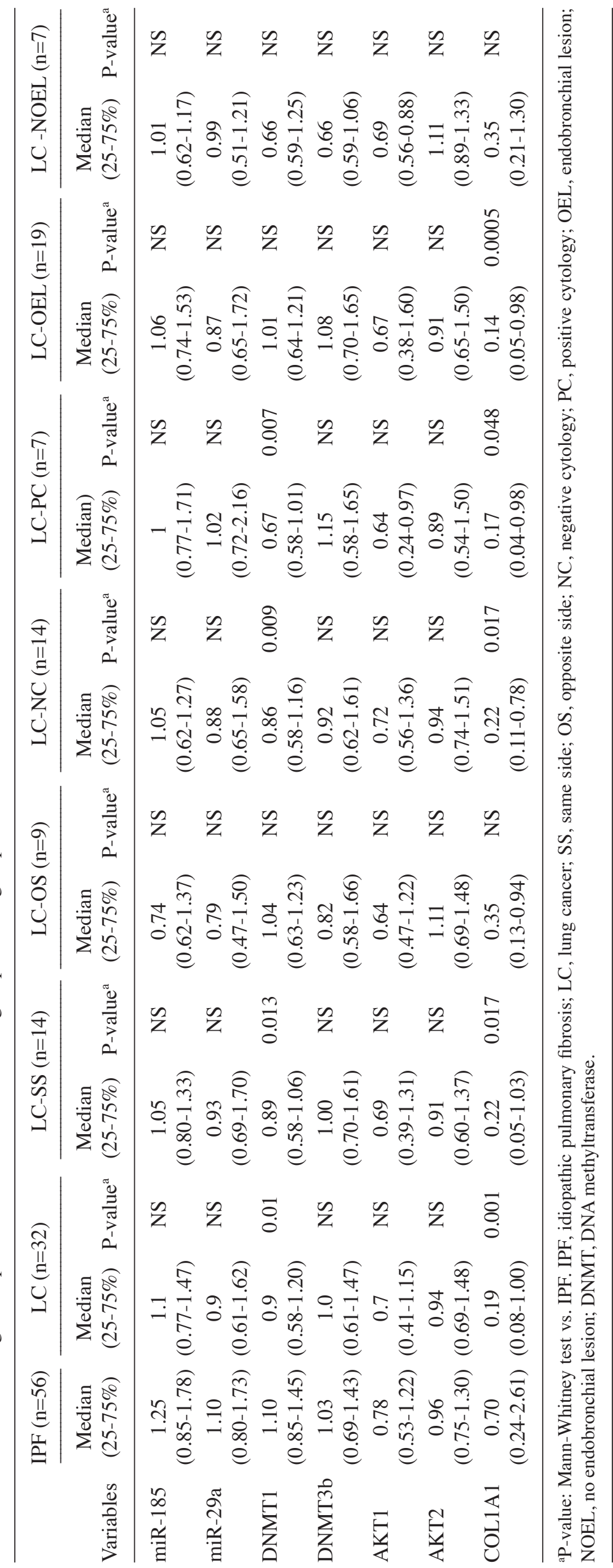


A

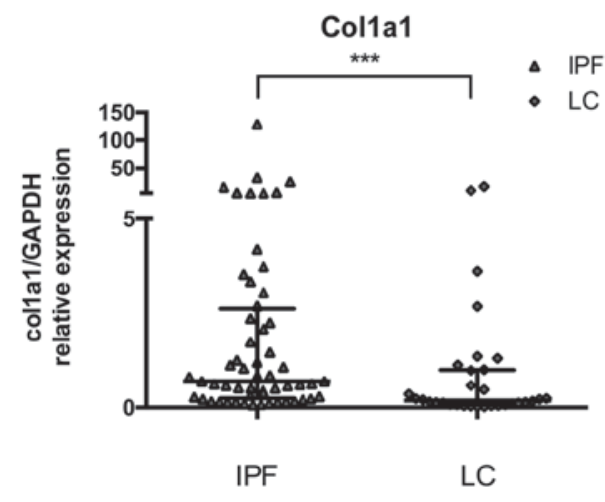

B

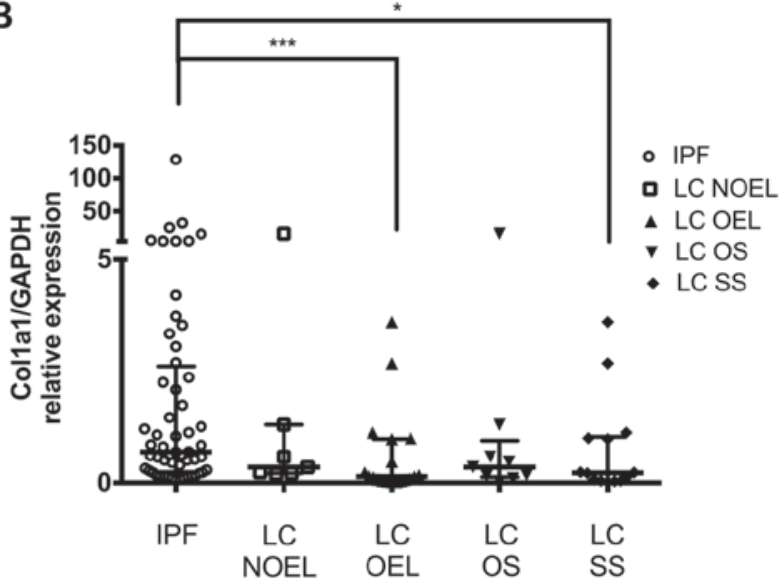

Figure 3. COL1A1 levels in LC patients compared to IPF patients. COL1A1 mRNA levels in BAL cells as measured by RT-qPCR and normalised using GAPDH (A) in the LC group and (B) in the LC subgroups NOEL, OEL, OS and SS as compared to IPF. ${ }^{*} \mathrm{P}<0.05,{ }^{* * *} \mathrm{P}<0.001, \mathrm{Mann}-\mathrm{Whitney}$ test. LC, lung cancer; IPF, idiopathic pulmonary fibrosis; BAL, bronchoalveolar lavage; NOEL, no endobronchial lesion; OEL, endobronchial lesion; OS, opposite side; SS, same side.

was noted previously. Assorting LC patients depending on the side the BAL procedure was performed and comparing them with IPF, further reduced levels of COL1A1 was detected in LC patients when the BAL procedure was done ipsilaterally of the lesion than contralaterally. The presence of an OEL during the bronchoscope signified more clearly reduced levels of COL1A1 (compared with IPF) than in the case of NOEL (Fig. 3A; Table III). Patients with the histological type of SCLC had COL1A1 levels similar with IPF levels, opposing the reduced levels in the NSCLC patients subgroup.

\section{Discussion}

This is a BAL study of the expression of major epigenetic molecules, miR-29a and miR-185, and their common targets DNMT1 and DNMT3b, involved in the DNA methylation process. The downregulation of these molecules has been previously connected with IPF and LC. The relationship between the lethal diseases which often coexist is an active field of research as common pathogenic pathways emerge, with major therapeutic implications. Our cardinal findings were: i) Both miR-185 and miR-29a were comparably expressed in IPF and LC BAL samples; ii) no direct correlation with miR-29a or miR-185 and their targets was observed, albeit DNMT1 downregulation was characteristic of LC and COL1A1 upregulation was representative of IPF BAL samples; and iii) in LC the malignant burden affected both DNMT1 and COL1A1 expression.

Similar levels of miR-29a and miR-185 were detected between IPF and LC in BAL cells while our previous findings showed that both miR-29a and miR-185 were downregulated in IPF relative to controls (25). The downregulation of miR-29a and miR-185 in LC tissue specimens has been previously established; however, to the best of our knowledge, this is the first study on LC BAL cells. Our results suggest that miR-185 is a novel common microRNA deregulation in IPF and LC next to previously identified microRNAs such as miR-29a. miR-29a and miR-185 expression showed a significant association in both IPF and LC BAL samples. A possible common regulation of the expression of the two microRNAs may be related to the increased levels of TGFb in both IPF and LC
BAL $(37,38)$. Activation of TGF- $\beta$ signaling and excessive accumulation of ECM proteins are observed in IPF and LC, highlighting a common molecular mechanism in both diseases that is directly linked to both microRNAs $(39,40)$. Of note, miRNA based-therapeutic strategies are already under evaluation for their use in several malignancies (41) and that imposes the need for in-depth study of similarities and differences between IPF and LC, focusing on key molecules involved in the multifarious function of alveolar macrophages.

For this reason, we examined the expression of a common target of miR-29a and miR-185 induced by $\mathrm{TGFb}$ and central fibrosis mediator collagen 1a in LC in comparison to IPF. Our previous results supported that the downregulation of miR-29a in IPF is associated with the overexpression of $C O L 1 A 1$ gene in BAL cells, confirming the active role of the miR-29a/COL1A1 pathway in AMs and lung tissue, while the expression profile of COL1A1 in LC has not been yet clarified. COL1A1 may be involved in carcinogenesis as aberrant expression levels were revealed in several malignancies including hepatocellular carcinoma (42), NSCLC tissue (43) and in malignant gastric tissue (44). Our findings, however, showed significantly increased levels of COL1A1 in IPF relative to LC in BAL cells that establishes the characteristic fibrotic profile of BAL cells in IPF, which appears to be lacking in LC BAL cells. The further reduced levels of COL1A1 accordingly with increased malignant burden cannot thus far be interpreted and further functional analysis of AMs in LC is needed.

A second pathway/axis affected by both miR-29a and miR-185 is the expression of DNMTs. An important concept currently put forward for the pathogenesis of IPF as in cancer is that common risk factors such as aging, cigarette smoke and environmental effects induce errors in the maintenance of the methylation marks of the genome creating aberrant DNA methylation patterns and accelerating the aging of our epigenome $(45,46)$. Global methylation profile in IPF lung tissue was different compared to the controls and partially similar to cancer $(6,7)$. Furthermore, an increased expression of the DNMTs was observed in both cancer and IPF lung tissue studies $(7,47)$, leading to site-specific hypermethylation and gene silencing. 
We have previously reported that DNMT1 mRNA levels in the BAL cells of IPF patients were similar to controls (25). In the current study, we observed a significant reduction in the mRNA levels of DNMT1 in LC BAL cells while, DNMT3b levels were similar in IPF and LC. DNMT1, in contrast to DNMT3, appears to function in cooperation with DNA damage repair pathways in order to maintain genomic stability and ablation or reduction of DNMT1 promotes mutagenic events (48), microsatellite instability and chromosomal translocations (49). Notably, the samples obtained near the malignant lesion or with positive malignant cell cytology results showed a more pronounced reduction of DNMT1 expression suggesting that reduced DNMT1 levels were associated with increased malignant burden.

LC is a common and prognostically determinant comorbidity among IPF patients. BAL procedure is a less invasive and harmless tool for revealing new, disease specific biomarkers regarding LC in IPF patients, for screening, risk stratification and diagnostic purposes, verging the promising concept of cancer liquid biopsy. It would be of great interest to study the expression profile of those miRNAs and their targets also in patients who simultaneously suffer from IPF and LC. MicroRNA based-therapeutic strategies are already under evaluation for their use in several malignancies (41) and IPF (50) and would greatly benefit from in depth study of similarities and differences between IPF and LC, focusing on key molecules involved in the multifarious function of alveolar macrophages.

Tissue specimens and cell lines are the predominant material of research; however, the role of BAL as a minimally invasive tool to study alveolar macrophages and their implication in pathogenesis continually recovers ground. In IPF alveolar macrophages deriving from monocytes recruited to the injured lungs are actively involved in the fibrotic process (51). Altered alveolar macrophage function in patients with LC has been recorded under the influence of tumor-associated polarizing events such as mediators and hypoxic tissue damage (52). Our study provides some insight with respect to common alveolar macrophage function in the two groups as demonstrated by the commonly reduced expression of miR-29a and miR-185. Further research is needed in order to identify BAL biomarkers for high risk patients and more targeted therapies.

\section{Acknowledgements}

The authors would like to thank the staff of the broncoscopy unit and the members of the pathology department of the University Hospital of Heraklion.

\section{Funding}

The project was funded by a grant from the Hellenic Thoracic Society (HTS 2012).

\section{Availability of data and materials}

The datasets used and/or analyzed during the current study are available from the corresponding author on reasonable request.

\section{Authors' contributions}

EB, ET and KMA conceived and designed the study. EB, ET, $\mathrm{CK}$ and TG performed the experiments. EB, ET, EV, GM and AT collected patient data. EB and ET performed statistical analysis. EB, ET and KMA wrote the manuscript. DAS, NT and KMA critically reviewed and edited the manuscript. All authors read and approved the manuscript and agree to be accountable for all aspects of the research in ensuring that the accuracy or integrity of any part of the work are appropriately investigated and resolved.

\section{Ethics approval and consent to participate}

The study was approved by the Ethics Committee of the University Hospital of Heraklion (IRB no. 17030/12-12-2013 for IPF patients and Reg. no. 140/4-2-2015 for LC patients). All the patients provided informed consent in written form.

\section{Consent for publication}

Not applicable.

\section{Competing interests}

Demetrios A. Spandidos is the Editor-in-Chief for the journal, but had no personal involvement in the reviewing process, or any influence in terms of adjudicating on the final decision, for this article.

\section{References}

1. Richeldi L, Collard HR and Jones MG: Idiopathic pulmonary fibrosis. Lancet 389: 1941-1952, 2017.

2. Antoniou KM, Hansell DM, Rubens MB, Marten K, Desai SR, Siafakas NM, Nicholson AG, du Bois RM and Wells AU: Idiopathic pulmonary fibrosis: Outcome in relation to smoking status. Am J Respir Crit Care Med 177: 190-194, 2008.

3. Selman M and Pardo A: Revealing the pathogenic and agingrelated mechanisms of the enigmatic idiopathic pulmonary fibrosis. an integral model. Am J Respir Crit Care Med 189: 1161-1172, 2014.

4. Giopanou I, Arendt KAM and Stathopoulos GT: Lung carcinogenesis and fibrosis taken together: Just coincidence? Curr Opin Pulm Med 23: 290-297, 2017.

5. Antoniou KM, Tomassetti S, Tsitoura E and Vancheri C: Idiopathic pulmonary fibrosis and lung cancer: A clinical and pathogenesis update. Curr Opin Pulm Med 21: 626-633, 2015.

6. Duruisseaux M and Esteller M: Lung cancer epigenetics: From knowledge to applications. Semin Cancer Biol S1044-579X(17)30166-9, 2017.

7. Sanders YY, Ambalavanan N, Halloran B, Zhang X, Liu H, Crossman DK, Bray M, Zhang K, Thannickal VJ and Hagood JS: Altered DNA methylation profile in idiopathic pulmonary fibrosis. Am J Respir Crit Care Med 186: 525-535, 2012.

8. Antoniou KM, Samara KD, Lasithiotaki I, Margaritopoulos GA, Soufla G, Lambiri I, Giannarakis I, Drositis I, Spandidos DA and Siafakas NM: Differential telomerase expression in idiopathic pulmonary fibrosis and non-small cell lung cancer. Oncol Rep 30: 2617-2624, 2013.

9. Hanahan D and Weinberg RA: Hallmarks of cancer: The next generation. Cell 144: 646-674, 2011.

10. Sarchianaki E, Derdas SP, Ntaoukakis M, Vakonaki E, Lagoudaki ED, Lasithiotaki I, Sarchianaki A, Koutsopoulos A, Symvoulakis EK, Spandidos DA, et al: Detection and genotype analysis of human papillomavirus in non-small cell lung cancer patients. Tumour Biol 35: 3203-3209, 2014. 
11. Samara KD, Antoniou KM, Karagiannis K, Margaritopoulos G, Lasithiotaki I, Koutala E and Siafakas NM: Expression profiles of Toll-like receptors in non-small cell lung cancer and idiopathic pulmonary fibrosis. Int J Oncol 40: 1397-1404, 2012.

12. Vancheri C: Idiopathic pulmonary fibrosis and cancer: Do they really look similar? BMC Med 13: 220, 2015.

13. Antoniou KM, Lasithiotaki I, Symvoulakis E, Derdas SP, Psaraki A, Spandidos DA, Stathopoulos EN, Siafakas NM and Sourvinos G: Molecular pathological findings of Merkel cell polyomavirus in lung cancer: A possible etiopathogenetic link? Int J Cancer 133: 3016-3017, 2013.

14. Lasithiotaki I, Antoniou KM, Derdas SP, Sarchianaki E, Symvoulakis EK, Psaraki A, Spandidos DA, Stathopoulos EN, Siafakas NM and Sourvinos G: The presence of Merkel cell polyomavirus is associated with deregulated expression of BRAF and $\mathrm{Bcl}-2$ genes in non-small cell lung cancer. Int J Cancer 133: 604-611, 2013.

15. Vassilakis DA, Sourvinos G, Spandidos DA, Siafakas NM and Bouros D: Frequent genetic alterations at the microsatellite level in cytologic sputum samples of patients with idiopathic pulmonary fibrosis. Am J Respir Crit Care Med 162: 1115-1119, 2000.

16. Tomassetti S, Gurioli C, Ryu JH, Decker PA, Ravaglia C, Tantalocco P, Buccioli M, Piciucchi S, Sverzellati N, Dubini A, et al: The impact of lung cancer on survival of idiopathic pulmonary fibrosis. Chest 147: 157-164, 2015.

17. Mizuno K, Mataki H, Seki N, Kumamoto T, Kamikawaji K and Inoue H: MicroRNAs in non-small cell lung cancer and idiopathic pulmonary fibrosis. J Hum Genet 62: 57-65, 2017.

18. Cushing L, Kuang PP, Qian J, Shao F, Wu J,Little F, Thannickal VJ, Cardoso WV and Lü J: miR-29 is a major regulator of genes associated with pulmonary fibrosis. Am J Respir Cell Mol Biol 45: 287-294, 2011.

19. Roderburg C, Urban GW, Bettermann K, Vucur M,Zimmermann H, Schmidt S, Janssen J, Koppe C, Knolle P, Castoldi M, et al: Micro-RNA profiling reveals a role for miR-29 in human and murine liver fibrosis. Hepatology 53: 209-218, 2011.

20. Patel V and Noureddine L: MicroRNAs and fibrosis. Curr Opin Nephrol Hypertens 21: 410-416, 2012.

21. Luna C, Li G, Qiu J, Epstein DL and Gonzalez P: Role of miR-29b on the regulation of the extracellular matrix in human trabecular meshwork cells under chronic oxidative stress. Mol Vis 15: 2488-2497, 2009.

22. Bian EB, Li J and Zhao B: miR-29, a potential therapeutic target for liver fibrosis. Gene 544: 259-260, 2014.

23. Cicchini C, de Nonno V, Battistelli C, Cozzolino AM, De Santis Puzzonia M, Ciafrè SA, Brocker C, Gonzalez FJ, Amicone L and Tripodi M: Epigenetic control of EMT/MET dynamics: HNF4 $\alpha$ impacts DNMT3s through miRs-29. Biochim Biophys Acta 1849: 919-929, 2015.

24. Wang Y, Zhang X, Li H, Yu J and Ren X: The role of miRNA-29 family in cancer. Eur J Cell Biol 92: 123-128, 2013.

25. Tsitoura E, Wells AU, Karagiannis K, Lasithiotaki I, Vasarmidi E, Bibaki E, Koutoulaki C, Sato H, Spandidos DA, Siafakas NM, et al: MiR-185/AKT and miR-29a/collagen 1a pathways are activated in IPF BAL cells. Oncotarget 7: 74569-74581, 2016.

26. Lei GS, Kline HL, Lee CH, Wilkes DS and Zhang C: Regulation of collagen $\mathrm{V}$ expression and epithelial-mesenchymal transition by miR-185 and miR-186 during idiopathic pulmonary fibrosis. Am J Pathol 186: 2310-2316, 2016.

27. Lu ZJ, Lu LG, Tao KZ, Chen DF, Xia Q, Weng JJ, Zhu F, Wang XP and Zheng P: MicroRNA-185 suppresses growth and invasion of colon cancer cells through inhibition of the hypoxia inducible factor- $2 \alpha$ pathway in vitro and in vivo. Mol Med Rep 10 2401-2408, 2014

28. Li S, Ma Y, Hou X, Liu Y, Li K, Xu S and Wang J: MiR-185 acts as a tumor suppressor by targeting AKT1 in non-small cell lung cancer cells. Int J Clin Exp Pathol 8: 11854-11862, 2015.

29. Qadir XV, Han C, Lu D, Zhang J and Wu T: miR-185 inhibits hepatocellular carcinoma growth by targeting the DNMT1/PTEN/Akt pathway. Am J Pathol 184: 2355-2364, 2014.

30. Ding X, Yu C, Liu Y, Yan S, Li W, Wang D, Sun L, Han Y, Li M, Zhang S, et al: Chronic obstructive sleep apnea accelerates pulmonary remodeling via TGF- $\beta / \mathrm{miR}-185 / \mathrm{CoLA} 1$ signaling in a canine model. Oncotarget 7: 57545-57555, 2016.

31. Xiao K, Luo X, Wang X and Gao Z: MicroRNA 185 regulates transforming growth factor $\beta 1$ and collagen 1 in hypertrophic scar fibroblasts. Mol Med Rep 15: 1489-1496, 2017.
32. Tang B, Li X, Ren Y, Wang J, Xu D, Hang Y, Zhou T, Li F and Wang L: MicroRNA-29a regulates lipopolysaccharide (LPS)-induced inflammatory responses in murine macrophages through the Akt1/ NF- $\kappa$ B pathway. Exp Cell Res 360: 74-80, 2017.

33. Li M, Li H, Liu X, Xu D and Wang F: MicroRNA-29b regulates TGF- $\beta 1$-mediated epithelial-mesenchymal transition of retinal pigment epithelial cells by targeting AKT2. Exp Cell Res 345: 115-124, 2016.

34. Park S, Hur JY, Lee KY, Lee JC, Rho JK, Shin SH and Choi CM: Assessment of EGFR mutation status using cell-free DNA from bronchoalveolar lavage fluid. Clin Chem Lab Med 55: 1489-1495, 2017.

35. Raghu G: Idiopathic pulmonary fibrosis: Guidelines for diagnosis and clinical management have advanced from consensus-based in 2000 to evidence-based in 2011. Eur Respir J 37: 743-746, 2011.

36. Travis WD, Brambilla E, Nicholson AG, Yatabe Y, Austin JHM, Beasley MB, Chirieac LR, Dacic S, Duhig E, Flieder DB, et al: The 2015 World Health Organization Classification of lung tumors: Impact of genetic, clinical and radiologic advances since the 2004 classification. J Thorac Oncol 10: 1243-1260, 2015.

37. Chen Z, Xu Z, Sun S, Yu Y, Lv D, Cao C and Deng Z: TGF- $\beta 1$, IL-6, and TNF- $\alpha$ in bronchoalveolar lavage fluid: Useful markers for lung cancer? Sci Rep 4: 5595, 2014.

38. Kumar RK, O'Grady R, Maronese SE and Wilson MR: Epithelial cell-derived transforming growth factor-beta in bleomycin-induced pulmonary injury. Int J Exp Pathol 77: 99-107, 1996.

39. Fernandez IE and Eickelberg O: The impact of TGF- $\beta$ on lung fibrosis: From targeting to biomarkers. Proc Am Thorac Soc 9: 111-116, 2012.

40. Eser PO and Jänne PA: TGF $\beta$ pathway inhibition in the treatment of non-small cell lung cancer. Pharmacol Ther: Nov 10, 2017 (Epub ahead of print).

41. Shah MY, Ferrajoli A, Sood AK, Lopez-Berestein G and Calin GA: microRNA therapeutics in cancer - An emerging concept. EBioMedicine 12: 34-42, 2016.

42. Hayashi M, Nomoto S, Hishida M, Inokawa Y, Kanda M, Okamura Y, Nishikawa Y, Tanaka C, Kobayashi D, Yamada S, et al: Identification of the collagen type $1 \alpha 1$ gene (COL1A1) as a candidate survival-related factor associated with hepatocellular carcinoma. BMC Cancer 14: 108, 2014.

43. Oleksiewicz U, Liloglou T, Tasopoulou KM, Daskoulidou N, Gosney JR, Field JK and Xinarianos G: COL1A1, PRPF40A, and UCP 2 correlate with hypoxia markers in non-small cell lung cancer. J Cancer Res Clin Oncol 143: 1133-1141, 2017.

44. Li J, Ding Y and Li A: Identification of COL1A1 and COL1A2 as candidate prognostic factors in gastric cancer. World J Surg Oncol 14: 297, 2016.

45. Selman M and Pardo A: Stochastic age-related epigenetic drift in the pathogenesis of idiopathic pulmonary fibrosis. Am J Respir Crit Care Med 190: 1328-1330, 2014.

46. Langevin SM, Pinney SM, Leung YK and Ho SM: Does epigenetic drift contribute to age-related increases in breast cancer risk? Epigenomics 6: 367-369, 2014.

47. Lin RK, Hsu HS, Chang JW, Chen CY, Chen JT and Wang YC: Alteration of DNA methyltransferases contributes to $5^{\prime} \mathrm{CpG}$ methylation and poor prognosis in lung cancer. Lung Cancer 55: 205-213, 2007

48. Jin B and Robertson KD: DNA methyltransferases, DNA damage repair, and cancer. Adv Exp Med Biol 754: 3-29, 2013.

49. Chen RZ, Pettersson U, Beard C, Jackson-Grusby L and Jaenisch R: DNA hypomethylation leads to elevated mutation rates. Nature 395: 89-93, 1998.

50. Cushing L, Kuang P and Lü J: The role of miR-29 in pulmonary fibrosis. Biochem Cell Biol 93: 109-118, 2015.

51. Misharin AV, Morales-Nebreda L, Reyfman PA, Cuda CM, Walter JM, McQuattie-Pimentel AC, Chen CI, Anekalla KR, Joshi N, Williams KJN, et al: Monocyte-derived alveolar macrophages drive lung fibrosis and persist in the lung over the life span. J Exp Med 214: 2387-2404, 2017.

52. Almatroodi SA, McDonald CF and Pouniotis DS: Alveolar macrophage polarisation in lung cancer. Lung Cancer Int 2014: 721087, 2014. 